\title{
Development of a consensus approach to upper limb rehabilitation early post stroke amongst a cohort of Western Australian therapists.
}

Jimena Garcia-Vega BPhty, Grad Dip in Neuro Rehab, Master of Neuro Rehab

Senior Physiotherapist, Department of Physiotherapy, Sir Charles Gairdner Hospital

Centre for Musculoskeletal Studies, School of Surgery, The University of Western Australia

Gillian Gregory BPhty

Acting Senior Physiotherapist, Department of Physiotherapy, Sir Charles Gairdner Hospital

Christopher RP Lind MBBS, FRACS

Consultant Neurosurgeon, Department of Neurosurgery, Sir Charles Gairdner Hospital. School of Surgery, The University of Western Australia

Barbara J Singer Dip PT, MSc (Health Sci), PhD, FACP

School of Surgery, The University of Western Australia

\section{ABSTRACT}

This study aimed to define commonly reported physiotherapy and occupational therapy terminology regarding rehabilitation interventions for moderate to severe upper limb deficits early post- stroke and to develop a consensus on 'standard' post-stroke upper limb rehabilitation across three stroke services. An audit was undertaken of all middle cerebral artery strokes admitted over a nine-month period to an acute tertiary hospital. Data were collected from 48 cases of middle cerebral artery stroke. Twenty four cases had moderate to severe upper limb impairment, and of these 16 were transferred to the two participating rehabilitation sites. A list of upper limb interventions documented in these 16 cases was distributed to therapists from the three participating sites (nine Physiotherapists, 13 Occupational Therapists) who subsequently attended focus groups. Definitions for reported interventions were developed, collated and refined until group agreement was reached using a modified Delphi method. Approaches to upper limb rehabilitation varied according to therapists' clinical experience and training background; however, definitions did not vary widely between services or disciplines. A consensus on 'usual care' for moderate to severe upper limb deficits within participating stroke services was developed from which a structured, individualised, impairment-based treatment template was produced for use in a subsequent interventional study.

\section{Garcia-Vega G, Gregory G, Lind C, Singer B (2016) Development of a consensus approach to upper limb rehabilitation early post stroke amongst a cohort of Western Australian therapists. New Zealand Journal of Physiotherapy 44(3): 133-147. doi: 10.15619/NZJP/44.3.03}

Key words: Stroke, Upper limb rehabilitation, Consensus

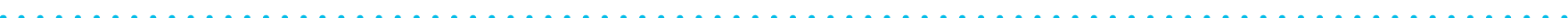

\section{INTRODUCTION}

According to the World Health Organisation (WHO 2015) stroke is the third most frequent cause of death and the leading cause of acquired adult disability in developed countries. About 15 million people suffer a stroke worldwide each year, and 5.5 million of these die while another 5 million are permanently disabled (World Stroke Organisation (2015).

The upper limb (UL) generally makes a poorer recovery poststroke than the lower limb (LL) (Kong et al 2011). Approximately $60 \%$ of patients with severe to complete UL paresis are unable to achieve full dexterity after 6 months post-stroke (Kwakkel et al 2003, van Kuijk 2009); while $71 \%$ of patients with mild to moderate initial UL paresis achieve some dexterity at 6 months and therefore have a significantly better prognosis for recovery (Nijland et al 2010). The most common impairments affecting UL function post-stroke are decreased motor control, spasticity, decreased sensation and proprioception, pain, decreased range of movement, motor dyspraxia, inattention/neglect, diplopia, homonymous hemianopia, and impaired cognition (Brewer et al 2013).

Several rehabilitation 'treatment taxonomies' have been developed to assist in standardising, prescribing and progressing therapy, dissemination of interventions, training of novice practitioners, interdisciplinary communication and the conduct and reporting of research (Arya et al 2012, Hart et al 2014, McDonnell et al 2013, Rosewilliam et al 2009, Whyte et al 2014). These include the evidence-based clinical algorithm to facilitate standardised intervention, prescription and progression for UL rehabilitation post-stroke, developed by McDonnell et al (2013); and a 'meaningful-task specific training (MTST) model' which outlines the use of a specific number of common tasks, incorporating unilateral and bilateral practice (Arya et al 2012). Both taxonomies have been shown to be feasible to guide UL therapy in subacute stroke care, as well as to encourage independent practice and increase the number of repetitions and time spent in therapy, which may facilitate achievement 
of the intensity of UL therapy recommended in the Australian and New Zealand Stroke Foundation stroke rehabilitation guidelines (Australian Stroke Foundation 2016, New Zealand Stroke Foundation 2016). These 'treatment taxonomies' add to a body of evidence that suggests that the content and intensity of UL rehabilitation can be standardised in a stroke population with various levels of impairment. Furthermore, it is feasible to implement such protocols in clinical practice and research studies (McDonnell et al 2013, Rosewilliam et al 2009, Wallace et al 2010).

In research trials of novel therapies the control condition is frequently described as 'usual care' without describing the actual intervention in sufficient detail to allow it to be replicated in a clinical setting. The literature has identified significant gaps in the reporting of non-pharmacological randomised controlled trials (RCT). In the extended Consolidated Standard of Reporting Trials (CONSORT) statement, Boutron et al (2008) provide a checklist of items to be reported in non-pharmacological trials, including a detailed description of interventions, procedures for individual tailoring of the intervention to participants according to their environment, details of how therapists' adherence with the treatment protocol(s) was monitored and provision of an explanation of any uncommon circumstances or modifications. Adherence to these guidelines can enable accurate recording and delivery of standardised interventions in non-pharmacological clinical trials.

This study was a preliminary phase of an RCT to explore noninvasive brain stimulation as an adjunct to UL rehabilitation postacute stroke (Garcia-Vega et al 2016). Hence, this qualitative descriptive study aimed to inform the development of a package of 'standard care' for the subsequent interventional trial and a recording tool to facilitate an accurate description of the treatment given to each individual participant by: (a) defining commonly reported physiotherapy and occupational therapy descriptors of approaches to UL rehabilitation post-stroke and (b) developing a consensus amongst therapists regarding 'usual care' in the management of moderate to severe UL deficits poststroke

\section{METHODS}

This study comprised four stages:

\section{Stage I: Retrospective notes audit}

The audit sought to accurately represent a cohort of patients admitted to three major Western Australian hospitals, where the subsequent pilot RCT was to be conducted. The intervention study only included first time ischaemic middle cerebral artery (MCA) territory strokes; therefore the audit was limited to this cohort. A report was generated including data on diagnosis, length of stay, and discharge destination from all neurological admissions with 'stroke-like' symptoms over a nine-month period at one acute tertiary centre. Diagnosis was subsequently verified from imaging reports via computed tomography (CT) and/or magnetic resonance imaging (MRI) and each case was categorised for stroke type (ischaemic or haemorrhagic, cortical or subcortical) and area of the brain affected. Medical records meeting the criteria were obtained and descriptions of physiotherapy and occupational therapy UL treatment during the inpatient admission of each case at the participating acute and rehabilitation centres were recorded. Treatment reports by all therapists (or allied health assistants) were screened to determine UL impairment severity and management during the acute phase of recovery and, for those with moderate to severe UL impairment, details of therapy were recorded. Where available, Chedoke McMaster Impairment Inventory (CMII) scores (Gowland et al 1993) were used to categorise patients' severity of UL impairment. Therapists' descriptive data were used to allocate impairment group when CMII scores were not available. A list of the most commonly documented interventions (more than $50 \%$ of patients receiving this treatment) in the acute and subacute rehabilitation settings was prepared from the audit data.

Stage II: Definition of UL interventions and consensus on "usual UL rehabilitation" in participating services A consensus on definitions of documented UL therapies provided to those audit cases who received both acute care and rehabilitation post-stroke in participating hospitals ( $n=16$ cases) was developed using a modified Delphi process (Hsu and Sandford 2007). All therapists at the acute stroke service and the two sites where the audit cases underwent rehabilitation were invited to participate in a focus group to discuss the audit data. All participants gave written consent to participate. Focus group meetings were held separately amongst physiotherapists and occupational therapists at each site. Audit results were presented to attendees, followed by a discussion of the list of UL treatment interventions identified from the file audit. All therapists were asked to prepare definitions of the terminology, and definitions were discussed and agreed upon as a group. Once recorded and collated, therapists were offered the opportunity to modify the list if they did not consider it was a true representation of current UL rehabilitation practices at their site (Round 1). Therapists who were identified in the audit but who were no longer working at the site were contacted by electronic mail to provide input into the process. The meeting was facilitated by the principal investigator, while another researcher recorded definitions and additional comments. Definitions of the terminology were collated into a consensus document which was returned to all therapists for comment or amendment until there was complete agreement that it was a true representation of the various types of UL therapy offered by their service for individuals receiving UL rehabilitation following moderate to severe stroke (Round 2).

Stage III: Development of UL therapy template for acute and subacute stroke rehabilitation

Collated site summaries and definitions were used to inform the development of an intervention template and glossary of UL therapies used in early stroke rehabilitation by the participating centres which could be considered to represent a 'usual care' UL therapy package at these services (Round 3).

Stage IV: Use of UL template as a recording tool in a pilot $\mathrm{RCT}$

A final version of the UL limb therapy template was utilised to inform individual therapy content, and to record the time allocated to each component as part of the subsequent interventional pilot RCT. 


\section{RESULTS}

Stage I: Retrospective notes audit

A total of 169 'stroke-like' cases were identified over the ninemonth period. Pathology from imaging reports (CT \& MRI) confirmed 10 diagnostic categories (Figure 1).

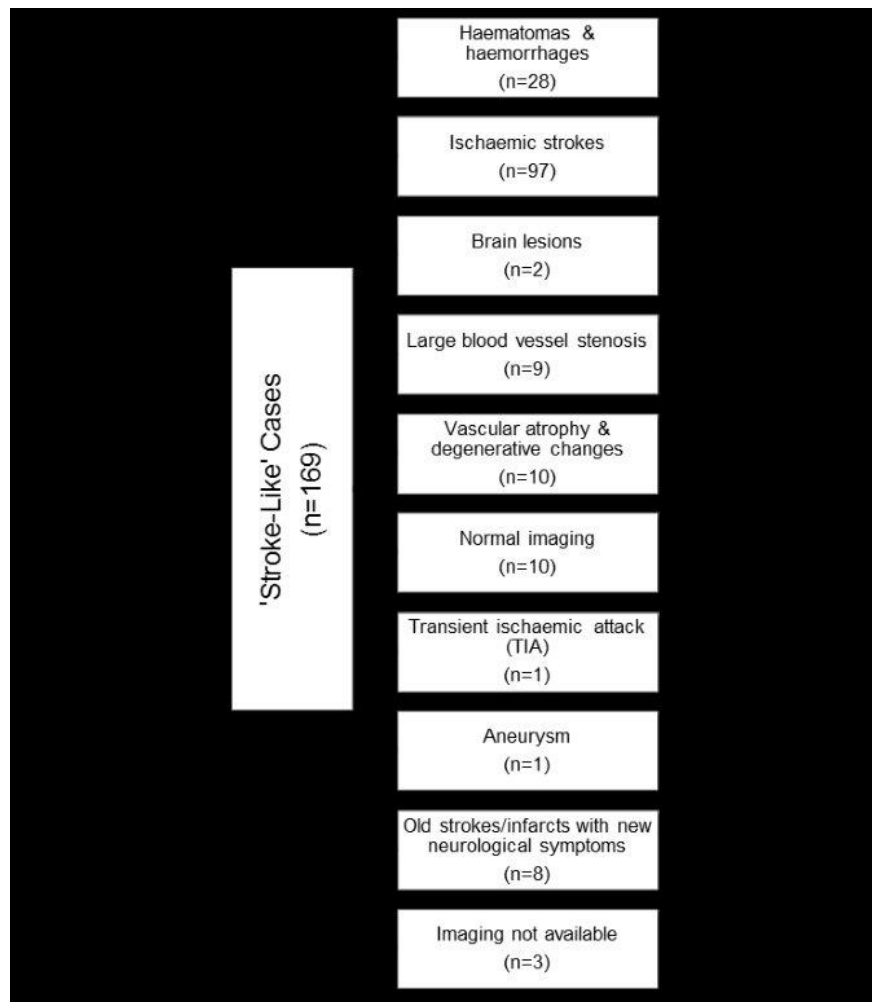

Figure 1: Retrospective notes audit 'stroke-like' diagnostic categories

Of the 97 ischaemic stroke cases identified, only 48 affected the MCA territory and/or a major MCA branch (ie $49.5 \%$ of total ischaemic stroke admissions) and these cases were included in the audit. It was determined that 22 patients had no UL impairment and two were sedated, therefore it was not possible to attain an accurate assessment of UL impairment. The 24 cases with UL impairment were classified into five categories according to CMII scores (Table 1).

Table 1: Upper limb impairment classification based on CMII scores $(n=24)$

\begin{tabular}{lc}
\hline Impairment Classification & $\begin{array}{c}\text { CMII Score Range } \\
\text { (Out of 7) }\end{array}$ \\
\hline Severe $(n=14)$ & $1-2$ \\
Moderate-Severe $(n=3)$ & $2-3$ \\
Moderate $(n=2)$ & $3-4$ \\
Mild-Moderate $(n=1)$ & $4-5$ \\
Mild $(n=4)$ & $5-7$ \\
\hline
\end{tabular}

Notes: CMII, Chedoke McMaster Impairment Inventory.

Only 16 cases out of the 24 identified in the acute service audit were transferred to participating rehabilitation sites, and therefore, only these cases were included in this review of UL management. Eleven of the 24 included cases went to rehabilitation service one (over 65 stroke service); and five patients went to rehabilitation service two (under 65 stroke service), the remaining eight cases were discharged to transitional care placement (TCP) $(n=1)$, Rehabilitation in the Home $(\mathrm{RITH})(\mathrm{n}=2)$, and home $(n=5)$. These cases were not included in this data set, as this study did not have ethics approvals for TCP and RITH and, therefore, medical records for rehabilitation in these cases were unable to be accessed. Audit data suggest that decisions to transfer patients to a rehabilitation facility were based primarily on impairment severity such as dense hemiplegia with motor and sensory components and global aphasia. Table 2 describes the demographics and UL impairment level on admission and discharge from the corresponding rehabilitation services of the 16 cases who were included in the audit.

Table 2: Demographic detail for the cases admitted to participating rehabilitation centres $(n=16)$ from whom treatment details were examined

\begin{tabular}{|c|c|c|c|c|c|c|c|}
\hline Patient & Age & Gender & CMIl Admission & $\begin{array}{l}\text { CMII } \\
\text { Discharge }\end{array}$ & Reason for rehabilitation & $\begin{array}{l}\text { LOS } \\
\text { (days) }\end{array}$ & $\begin{array}{l}\text { Final discharge } \\
\text { destination }\end{array}$ \\
\hline 1 & 78 & $\mathrm{~F}$ & $\begin{array}{l}\text { Arm: } 5 \text { Hand: } 5 \\
\text { Mild }\end{array}$ & $\begin{array}{l}\text { Arm: } 5 \text { Hand: } 5 \\
\text { Mild }\end{array}$ & UL rehab, Global aphasia & 33 & LLC (Hostel) \\
\hline 2 & 71 & M & $\begin{array}{l}\text { Arm: } 7 \text { Hand: } 6 \\
\text { Very Mild }\end{array}$ & $\begin{array}{l}\text { Arm: } 7 \text { Hand: } 6 \\
\text { Very Mild }\end{array}$ & UL rehab, HLB, visual imp & 5 & Home (ESD) \\
\hline 3 & 69 & M & $\begin{array}{l}\text { Arm: } 1 \text { Hand: } 1 \\
\text { Severe }\end{array}$ & $\begin{array}{l}\text { Arm: } 1 \text { Hand: } 2 \\
\text { Severe }\end{array}$ & $\begin{array}{l}\text { UL \& LL rehab, pusher } \\
\text { syndrome \& motor/sensory } \\
\text { neglect }\end{array}$ & 100 & $\begin{array}{l}\text { HLC (Residential } \\
\text { Institution) }\end{array}$ \\
\hline 4 & 85 & $\mathrm{~F}$ & $\begin{array}{l}\text { Arm: } 6 \text { Hand: } 6 \\
\text { Mild }\end{array}$ & $\begin{array}{l}\text { Arm: } 6 \text { Hand: } 6 \\
\text { Mild }\end{array}$ & $\begin{array}{l}\text { High level balance \& exercise } \\
\text { tolerance }\end{array}$ & 15 & Home (ESD) \\
\hline 5 & 65 & $\mathrm{~F}$ & $\begin{array}{l}\text { Arm: } 1 \text { Hand: } 1 \\
\text { Severe }\end{array}$ & $\begin{array}{l}\text { Arm: } 2 \text { Hand: } 2 \\
\text { Severe }\end{array}$ & UL rehab, sitting balance & 70 & $\begin{array}{l}\text { HLC (Transitional } \\
\text { Care Placement) }\end{array}$ \\
\hline
\end{tabular}




\begin{tabular}{|c|c|c|c|c|c|c|c|}
\hline 6 & 71 & M & $\begin{array}{l}\text { Arm: } 2 \text { Hand: } 2 \\
\text { Severe }\end{array}$ & $\begin{array}{l}\text { Arm: } 2 \text { Hand: } 2 \\
\text { Severe }\end{array}$ & $\begin{array}{l}\text { UL \& gait rehab \& high level } \\
\text { balance }\end{array}$ & 67 & $\begin{array}{l}\text { Home } \\
\text { (Home Link) }\end{array}$ \\
\hline 7 & 66 & $\mathrm{~F}$ & $\begin{array}{l}\text { Arm: } 1 \text { Hand: } 1 \\
\text { Severe }\end{array}$ & $\begin{array}{l}\text { Arm: } 1 \text { Hand: } 4 \\
\text { Severe-Moderate }\end{array}$ & $\begin{array}{l}\text { UL \& gait rehab, high level } \\
\text { balance }\end{array}$ & 59 & $\begin{array}{l}\text { Home } \\
\text { (RITH then } \\
\text { Stroke Clinic) }\end{array}$ \\
\hline 8 & 75 & $\mathrm{~F}$ & $\begin{array}{l}\text { Arm: } 7 \text { Hand: } 7 \\
\text { Nil Impairment }\end{array}$ & $\begin{array}{l}\text { Arm: } 7 \text { Hand: } 7 \\
\text { Nil Impairment }\end{array}$ & $\begin{array}{l}\text { High level balance /mobility, } \\
\text { gait rehab }\end{array}$ & 67 & Home \\
\hline 9 & 67 & M & $\begin{array}{l}\text { Arm: } 7 \text { Hand: } 6 \\
\text { Very Mild }\end{array}$ & $\begin{array}{l}\text { Arm: } 7 \text { Hand: } 7 \\
\text { Nil Impairment }\end{array}$ & UL rehab (fine motor skills) & 14 & Home (ESD) \\
\hline 10 & 75 & $\mathrm{~F}$ & $\begin{array}{l}\text { Arm: n/a Hand: n/a } \\
\text { Mild }\end{array}$ & $\begin{array}{l}\text { Arm: n/a Hand: n/a } \\
\text { Very Mild }\end{array}$ & UL rehab & 30 & Home (ESD) \\
\hline 11 & 78 & $\mathrm{~F}$ & $\begin{array}{l}\text { Arm: } 7 \text { Hand: } 7 \\
\text { Nil Impairment }\end{array}$ & $\begin{array}{l}\text { Arm: } 7 \text { Hand: } 7 \\
\text { Nil Impairment }\end{array}$ & Cognitive rehab & 1 & Home (ESD) \\
\hline 12 & 60 & M & $\begin{array}{l}\text { Arm: } 2 \text { Hand: } 2 \\
\text { Severe }\end{array}$ & $\begin{array}{l}\text { Arm: } 2 \text { Hand: } 2 \\
\text { Severe }\end{array}$ & $\begin{array}{l}\text { UL \& LL rehab (motor \& } \\
\text { sensory) }\end{array}$ & 122 & $\begin{array}{l}\text { Home } \\
\text { (RITH then } \\
\text { outpatients) }\end{array}$ \\
\hline 13 & 51 & M & $\begin{array}{l}\text { Arm: } 2 \text { Hand: } 2 \\
\text { Severe }\end{array}$ & $\begin{array}{l}\text { Arm: } 2 \text { Hand: } 2 \\
\text { Severe }\end{array}$ & $\begin{array}{l}\text { UL \& LL rehab (motor \& } \\
\text { sensory), gait rehab, high } \\
\text { level balance }\end{array}$ & 135 & Unknown \\
\hline 14 & 59 & $\mathrm{~F}$ & $\begin{array}{l}\text { Arm: } 1 \text { Hand: } 1 \\
\text { Severe }\end{array}$ & $\begin{array}{l}\text { Arm: } 1 \text { Hand: } 1 \\
\text { Severe }\end{array}$ & UL \& LL rehab (motor) & 32 & $\begin{array}{l}\text { Rehab centre in } \\
\text { New Zealand }\end{array}$ \\
\hline 15 & 64 & M & $\begin{array}{l}\text { Arm: } 1 \text { Hand: } 2 \\
\text { Severe }\end{array}$ & $\begin{array}{l}\text { Arm: } 2 \text { Hand: } 2 \\
\text { Severe }\end{array}$ & UL \& LL rehab (motor) & 114 & $\begin{array}{l}\text { Home } \\
\text { (RITH then } \\
\text { outpatients) }\end{array}$ \\
\hline 16 & 58 & $\mathrm{~F}$ & $\begin{array}{l}\text { Arm: } 2 \text { Hand: } 2 \\
\text { Severe }\end{array}$ & $\begin{array}{l}\text { Arm: } 2 \text { Hand: } 2 \\
\text { Severe }\end{array}$ & $\begin{array}{l}\text { UL \& LL rehab (motor \& } \\
\text { sensory), gait rehab and high } \\
\text { level balance }\end{array}$ & 92 & $\begin{array}{l}\text { Home } \\
\text { (RITH then } \\
\text { outpatients) }\end{array}$ \\
\hline
\end{tabular}

Notes: CMII, Chedoke McMaster Impairment Inventory; M, male; F, female; LLC, low level care; LOS, length of Stay; HLC, high level care; ESD, Early Supported Discharge; RITH, Rehabilitation in The Home; UL, upper limb; LL, lower limb; n/a, not applicable.

Across the two disciplines, the six most common interventions in the acute rehabilitation setting (reported in more than $50 \%$ of cases) were: UL facilitation, sensory input, 'trunk work', ADL retraining, UL positioning and passive range of motion (PROM). Overall, physiotherapists incorporated more active and active-assisted interventions into their UL treatment compared to occupational therapists. It was noted that in the acute setting, physiotherapists focused on facilitating any UL or trunk movement, whilst in rehabilitation settings, therapists were more focused on task-specific practice such as reach and grasp and other fine motor skills - although facilitatory approaches were still utilised. Occupational therapists in both settings prioritised ADL retraining. Acute care occupational therapists focused their interventions on self-care tasks such as hair combing, tooth brushing and dressing tasks; whilst in rehabilitation therapists opted for tasks involving higher executive functions such as cooking, shopping, community access, return to driving and home discharge planning. The tasks chosen were age appropriate: for instance, workshop classes (for employment related activities) were offered in the younger rehabilitation setting.

Both groups used 'sensory input/re-training' but this was documented much more frequently in the treatment records of physiotherapists than occupational therapists. Reach and grasp practice was only documented in about a third of the cases (physiotherapists 42\%, and occupational therapists $29 \%$ ) and education was more likely to be provided by occupational therapists than physiotherapists. The most commonly documented interventions in the acute and subacute rehabilitation settings are listed in Table 3 for physiotherapy and Table 4 for occupational therapy. 
Table 3: Most frequently delivered physiotherapy interventions in the acute and sub-acute rehabilitation settings

\begin{tabular}{lc}
\hline Acute Rehabilitation & $\begin{array}{c}\text { Frequency of } \\
\text { Documentation (\%) }\end{array}$ \\
\hline Facilitation of Fractionated Movement & 87.5 \\
Sensory input / Retraining & 58.3 \\
Trunk Activation / Facilitation & 50 \\
\hline Sub-Acute Rehabilitation & Frequency of \\
Documentation (\%)
\end{tabular}

Notes: UL, upper limb; PROM, passive range of motion.

Table 4 : Most frequently delivered occupational therapy interventions in the acute and sub-acute rehabilitation settings

\begin{tabular}{lc}
\hline Acute Rehabilitation & $\begin{array}{c}\text { Frequency of } \\
\text { Documentation (\%) }\end{array}$ \\
\hline ADL Retraining & 92 \\
Positioning & 83.3 \\
PROM / Ranging & 75 \\
\hline Sub-Acute Rehabilitation & Frequency of \\
Documentation (\%)
\end{tabular}

Notes: ADL, activities of daily living; UL, upper limb; PROM, passive range of motion.
Stage II: Definition of UL interventions and consensus on 'usual UL rehabilitation' in participating services (Rounds 1 and 2)

Twenty two female therapists, comprising nine physiotherapists and 13 occupational therapists, participated in a total of five focus groups. The participating therapists' level of experience ranged from 10 months to 39 years in neurological rehabilitation. The average focus group duration was one hour per discipline at their corresponding sites. It is important to note that some of the participants in the focus groups were not the same therapists whose notes were audited due to staff rotating out of area. Two therapists - one no longer working at the same facility, the other on leave - participated in the modified Delphi process via electronic mail.

Definitions were reviewed twice by all therapists prior to reaching a final consensus. Agreed definitions for each intervention are provided in Appendix 1. For the most part, the terminology used in treatment notes was defined very similarly by both discipline groups. Unsurprisingly, theoretical knowledge underpinning treatment interventions was greater according to level of seniority and experience in the field of neurological rehabilitation. Therapists' treatment approach also varied due to their training background.

The data from the audit reflected a set of cases from two years prior to the focus groups; consequently, it was necessary to determine if they still represented the most commonly used UL interventions currently provided by each service. Therapists were also given the opportunity to add or change any of the intervention definitions, to represent their current practice and understanding. For instance, therapists opined that the term 'trunk work' was too general and inaccurate; they suggested 'trunk activation/facilitation' be used to describe this intervention. Other terms such as 'sensory bombardment' were also amended to 'sensory input/re-training'. Functional interventions were described in more detail than ' $A D L$ retraining'; and more complex tasks such as 'bimanual tasks or activities' were included. Further elaboration was also given about the nature of specific "Bobath" interventions documented in the file audit.

Although time spent treating the UL was not able to be reported from the file audit, it was considered by group members that on average, patients received daily treatment of approximately 30 minutes duration per discipline for 5 days per week. These 30-minute sessions included assessments and all UL and LL therapy. Therapists were unable to quantify how much time they spent on average on UL specific therapy; however, they did express that it was likely to be minimal in the acute care facility.

Stage III: Development of UL therapy template in acute and subacute stroke rehabilitation (Round 3)

A template was developed from the data gathered from the audit and participants' additions and suggestions were incorporated in order to reflect current practice in early stroke rehabilitation at the participating centres (Appendix 2). The focus group discussions also allowed for the development of an accompanying glossary of definitions (Appendix 1).

The template outlines a repertoire of impairment-based interventions that may be used in the management of 
individuals with moderate to severe UL impairment in the acute and subacute stages of rehabilitation post-stroke. The interventions are categorised as 'passive', 'active-assisted' or 'active' which may incorporate practice of a functional task. These commonly used terminologies were also categorised under sensory and motor specific interventions; as well as those addressing other impairments such as management of tone, oedema and inattention/neglect. Functional tasks were primarily categorised under 'ADL specific' and 'reach and grasp' tasks. Finally, other interventions less commonly reported were included such as splinting, handwriting skills, 'workshop' and mirror box therapy (Appendix 2).

Stage IV: Use of UL template as a recording tool in a pilot RCT

The template was tested for ease of use and feasibility of implementation in a pilot RCT where it was used to direct and record care within a standard set of choices applying to moderate to severe UL impairment (Garcia-Vega et al 2016). It was shown to be an efficient recording tool providing an accurate description of the treatment given to participants in the interventional trial; as well as facilitating documentation of the treatment for both research records and patient clinical handover between therapists.

\section{DISCUSSION}

The purpose of this study was to document common practices in UL rehabilitation within a group of acute and subacute stroke services and to achieve an agreement on definitions of treatment to inform the development of a standardised template of 'usual care' UL rehabilitation post-acute stroke for use in a subsequent interventional pilot study. This template was not intended to provide the most evidence-based or 'best practice' UL interventions, but rather to reach a consensus on care provided amongst a small group of stroke units in Perth, Western Australia.

The literature states that there is inadequate reporting of interventions in pilot and feasibility studies, as well as phase II RCT studies; and that researchers need to adhere to guidelines provided to describe a package of 'usual care' such as the extended CONSORT statement (Boutron et al 2008) and the 2010 CONSORT (Schulz et al 2010) update. Such standardised processes are necessary in order to ensure high quality research that characterises the control treatment as well as the novel one.

The template developed from the consensus process in this study was utilised to plan and document the 'usual care' component of treatment in a safety and feasibility pilot RCT of non-invasive brain stimulation and UL rehabilitation post-acute stroke (Garcia-Vega et al 2016). This approach to standardising and documenting current rehabilitation practices did cause feelings of unease amongst some of the participating therapists, as their notes/documentation were audited by the principal investigator who is also one of their peers. This was one reason why focus group sessions were held separately for the three sites involved and for the two disciplines. However, it was made clear to participants that the rationale behind developing a 'standard of care' template was not to assess current practice against evidence based guidelines, but to achieve a consensus on a standard UL 'therapy package' to be delivered by a research therapist, which would be acceptable to the clinicians whose patients were involved in the subsequent interventional pilot study.

This process of involving clinicians in the consensus development allowed therapists to have confidence that the treatment given during the intervention phase was similar to that which they would have provided to their patients. This helped to gain therapists' compliance with non-treatment of the UL during the subsequent interventional study, which preserved the integrity of the intervention protocol, as per recommendations by Boutron et al (2008) and Schulz et al (2010).

Protocol differences exist between the way the template reported here was developed and previous literature. For instance, the template described by McHugh et al (2014) sourced rehabilitation interventions from a much wider population of therapists via a national survey in the UK; while the present study focused on data from one metropolitan acute and two subacute stroke services. Similarly to the UL therapy protocol developed by Rosewilliam et al (2009), the UL template developed from this study was based on an audit of UL interventions in stroke rehabilitation, which were categorised as passive, active assisted, and active. This categorisation allowed clear and concise documentation of intervention provided. However, Rosewilliam et al's (2009) protocol provides more specific guidance on progression of treatment than the current UL rehabilitation template, which was intended to offer an array of options, so that the research therapists could use their clinical judgement to guide the provision of individualised rehabilitation programmes.

Other authors have taken a different approach to the development of treatment templates. An example is McDonnell et al's (2013) evidence-based clinical algorithm, which standardises prescription and progression of UL interventions for people following stroke. This algorithm is structured around 18 critical impairments and covers a range of five domains: sensation, passive range of movement, strength, unilateral and bilateral dexterity. The therapists who contributed to the current template identified similar impairments and domains as McDonnell et al (2013). Similarly, the national survey of UK stroke rehabilitation practice by McHugh et al (2014) reported five main treatment categories, ranging from passive to most active, and was even inclusive of assistive technologies.

Other 'treatment taxonomies' have standardised rehabilitation approaches beyond therapy content, such as the treatment protocol developed by Wallace et al (2010) that provides a standardised intensity-based prescription of UL rehabilitation in a cohort of individuals with chronic stroke. Wallace et al (2010) demonstrated that UL therapy can also be standardised in relation to intensity, and can be goal-orientated and tailored regardless of stroke severity. This approach allowed the implementation of individualised rehabilitation, which was well tolerated by patients and therapists, and was feasible to administer in a multisite trial. Similarly, in the current study, the standardised template was found to be feasible to implement in a multisite clinical trial. It provided an array of interventions suitable for patients with moderate to severe sensorimotor UL impairments, and specifically tailored to patients in the acute and subacute stages of rehabilitation post-stroke. One caveat is 
that the protocol developed by Wallace et al (2010) was based on a chronic stroke cohort, and may not be suitable for use in acute stroke rehabilitation. For instance, the protocol requires one hour of therapy per day for 10 consecutive working days. Based on feedback from the participants in this study, anything longer than 30-minute sessions in an acute stroke population may have not have been feasible to administer due to patient tolerance and staffing issues.

The main variations of the treatment descriptors recorded in the present study appeared to be due to the level of experience, training background and knowledge of individual clinicians, both inter- and intra-professionally. Some therapists were more biased towards practice based on the Bobath concept (British Bobath Association 2009), while other therapists referred predominantly to the Motor Re-learning approach (Carr and Shepherd 2010); hence their descriptions were more reflective of the task specific practice model. There were some discipline specific differences. For instance, physiotherapists were more likely to approach facilitation of UL movement and trunk activation proximally using techniques such as 'scapular setting' (see Appendix 1 for a detailed definition); conversely, the occupational therapists most commonly approached treatment of poor trunk control via activation of the hand, for instance involving tasks such as reach and grasp retraining. Some therapists included a lot of focus on regaining trunk and pelvic control prior to addressing the UL deficits, whilst others approached UL retraining within the context of a functional task. These opposing viewpoints were a point of discussion amongst therapists, some of whom recognised that the Bobath terminology documented was not contemporary with recent descriptions (British Bobath Association 2009). There were discrepancies between same site therapists (occupational therapists versus physiotherapists) in regard to description of practices such as sensory re-training, facilitation of movement (proximal versus distal), and postural sets. In general, physiotherapists were more familiar with the Bobath approach than occupational therapists. Despite these variations it was possible to get agreement on definitions of the documented interventions amongst therapists in all participating services.

There were a number of limitations on the present study. The UL template was not intended to be based on 'best practice' or 'most evidence based care' but on what was 'usual care' in the participating services as described by focus group participants. Consequently, it does not necessarily equate with recommendations from international guidelines for management of the hemiplegic UL. However, clinicians involved in the consensus development have suggested that this template could be used to guide students and novice clinicians, as well as facilitating the delivery of research interventions. This template is not intended to be a representation of the practice of all therapists working in stroke services in the Perth metropolitan area where data were collected, nor indeed across other stroke services in Western Australia, as it may be limited by particular features of the practice of the participating therapists. Although these agreed definitions may be useful to other therapists trying to describe 'usual care' in their practice, this was not the intended purpose of the template.
In summary, UL rehabilitation after stroke cannot be a 'one size fits all' recipe; it must be tailored to stage of recovery, severity of impairment and individual patient factors. Previous UL rehabilitation paradigms have been informed by audits, national surveys or summaries of evidence-based practice, with different protocols providing progression options, ways to promote therapy intensity or an array of interventions categorised from passive, active assisted and active (Arya et al 2012, McDonnell et al 2013, McHugh et al 2014, Rosewilliam et al 2009). Current CONSORT guidelines (Boutron et al 2008, Schulz et al 2010) also provide a framework for reporting both control and novel interventions in non-pharmacological research studies. In the present study, a UL intervention template was developed based on a file audit and subsequent discussion amongst therapists to agree on definitions and practice that comprised 'usual care' in their services. It provided a framework of current and realistic UL rehabilitation practice at the participating acute and rehabilitation facilities in the one metropolitan area, which was subsequently successfully used to inform and document 'usual care' in an interventional trial of the addition of non-invasive brain stimulation to UL rehabilitation in acute stroke (GarciaVega et al 2016)

\section{CONCLUSION}

The template that has been developed from this study provides a structured impairment-based approach that could allow therapists to individualise their treatments within a set of welldefined interventions including development of functional skills, specific task-practice and application of manual techniques. This template has the potential to be used to inform a 'standard package of care' for rehabilitation of moderate to severe UL deficits post-stroke, in addition to providing a standardised recording tool in clinical research trials, which may facilitate accurate and time efficient documentation and replication of the care provided across services.

\section{KEY POINTS}

1. Common practices in UL rehabilitation (acute and sub-acute) were explored amongst therapists from a small group of acute and rehabilitation stroke services. The main findings were: (1) terminology used was defined very similarly by both physiotherapy and occupational therapy discipline groups; and (2) differences in treatment focus amongst therapists appeared to be due to the degree of experience and training background of individual clinicians, both inter- and intraprofessionally.

2. Across the continuum of care, physiotherapists tended to use more active interventions such as facilitation of fractionated movement, sensory / proprioceptive input, task re-training, trunk activation/facilitation and joint mobilisation (hands and shoulder), whilst occupational therapists in the acute setting reported more passive interventions such as PROM and UL positioning, with functional task-oriented retraining becoming the focus in the subacute stages of rehabilitation.

3. The template developed in this study provides a concise and easy to use tool to guide and document rehabilitation for those with moderate to severe UL deficits in the acute and subacute stages post-stroke. 


\section{PERMISSIONS}

Ethical approval was granted by the Sir Charles Gairdner Hospital (SCGH) and Osborne Park Hospital (OPH) Human Research Ethics Committee (trial number 2012-127); reciprocal ethics approvals from Royal Perth Hospital (RPH) (reference number REG 12-021), and The University of Western Australia (reference number RA/4///6040) were also obtained.

All focus group participants have given their permission to be named in the acknowledgements.

\section{DISCLOSURES}

This research was funded by the Sir Charles Gairdner and Osborne Park Health Care Group Research Advisory Committee (RAC Grant 2012/13 - Project No: HREC 2012-127), the Sir Charles Gairdner Research Foundation and the Sir Charles Gairdner Hospital Physiotherapy Department.

I declare on behalf of myself and the other authors that we know of no competing interests (financial, professional or personal) which may be perceived to interfere with or bias any stage of the writing or publication process. This includes, but is not restricted to, any factors that may influence full and objective presentation of the article, peer review and editorial decisions.

\section{ACKNOWLEDGEMENTS}

We gratefully acknowledge all the therapists who contributed to the focus groups: Alisha Anderson, Tracy Beckwith, Sarah Bennett, Anya Calame, Danielle Carvalho, Courtney Colliss (nee Janzen), Leanne Cormack, Ashlea Dichiera, Laura Ensov, Emma Jane Hill, Sascha Holbrook, Joanne Jenkins (nee Fisher), Kimberly Keeley Kate Kruger, Jaye Lange, Jessica Nolan, Sarah Rose, Ellen Sean-Ducrow, Karen Smith, Claire Tucak, Jemma Vyse, and Jocelyn White.

\section{ADDRESS FOR CORRESPONDENCE}

Professor Barbara Singer

School of Surgery, M509, Faculty of Medicine, Dentistry and Health Science, The University of Western Australia, 35 Stirling Highway, Crawley, 6009, Western Australia.

Email: barbara.singer@uwa.edu.au

\section{REFERENCES}

Australian Stroke Foundation (2016) https://strokefoundation.com.au [Accessed January, 21st, 2016].

Arya K, Verma R, Garg R, Sharma M, Agarwal M, Aggarwal G (2012) Meaningful task-specific training (MTST) for stroke rehabilitation: a randomised controlled trial. Topics in Stroke Rehabilitation 19: 193-211. doi: 10.1310/tsr1903-193.

Bobath Concept:Theory and Clinical Practice in Neurological Rehabilitation (2009). West Sussex: Wiley-Blackwell.

Boutron I, Moher D, Altman DG, Schulz KF, Ravaud P (2008) Extending the CONSORT statement to randomized trials of non-pharmacologic treatment: explanation and elaboration. Annals of Internal Medicine 148: 295-309.

Brewer L, Horgan F, Hickey A, Williams D (2013) Stroke rehabilitation: recent advances and future therapies. Quarterly Journal of Medicine 106 11-25. doi: 10.1093/qjmed/hcs174.
Carr J, Shepherd R (2010) Neurological Rehabilitation: Optimizing Motor Performance $\left(2^{\text {nd }}\right.$ edn). London: Churchill Livingstone.

Garcia-Vega J, Gregory G, Lind CPR, Blacker D, Ghosh S, Cooper I, Singer BJ (2016) Safety and feasibility of the application of cathodal transcranial direct current stimulation plus upper limb therapy in acute stroke. Asia Pacific Stroke Conference 2016. Abstracts of the Annual Conference of the Asia Pacific Stroke Organization (APSO) Combined with Stroke Society of Australasia, Brisbane, Qld., Australia, July 14-17, 2016: Abstracts. Cerebrovascular Diseases 2016;42(suppl 1):1-157.

Gowland C, Stratford P, Ward M, Moreland J, Torresin W, Van Hullenaar S, Sandford J, Barreca S, Vanspall B, Plews N (1993) Measuring physical impairment and disability with the Chedoke-McMaster stroke assessment. Stroke 24: 58-63.

Hart T, Tsaousides T, Zanca J, Whyte J, Packel A, Ferraro M, Dijkers M (2014) Toward a theory-driven classification of rehabilitation treatments. Archives of Physical Medicine and Rehabilitation 95: 33-44. doi: 10.1016/j. apmr.2013.05.032.

Hsu C-C, Sandford BA (2007) The Delphi technique: making sense of consensus. Practical Assessment, Research \& Evaluation 12: 1-8.

Kong K, Chua K, Lee J (2011) Recovery of upper limb dexterity in patients more than 1 year after stroke: frequency, clinical correlates and predictors. NeuroRehabilitation 28: 105-111. doi: 10.3233/NRE-2011-0639.

Kwakkel G, Kollen B, van der Grond J, Prevo A (2003) Probability of regaining dexterity in the flaccid upper limb: impact of severity of paresis and time since onset in acute stroke. Stroke 34: 2181-2186.

McDonnell M, Hillier S, Esterman A (2013) Standardizing the approach to evidence-based upper limb rehabilitation after stroke. Topics in Stroke Rehabilitation 20: 432-440. doi: 10.1310/tsr2005-432

McHugh G, Swain I, Jenkinson D (2014) Treatment components for upper limb rehabilitation after stroke: a survery of UK national practice. Disability and Rehabilitation: 36:925-31. doi: 10.3109/09638288.2013.824034.

New Zealand Stroke Foundation (2016) http://stroke.org.nz [Accessed January, 21 $\left.1^{\text {st }}, 2016\right]$.

Nijland R, van Wegen E, Harmeling-van der Wel B, Kwakkel G (2010) Presence of finger extension and shoulder abduction within 72 hours after stroke predicts functional recovery: early prediction of functional outcome after stroke: the EPOS cohort study. Stroke 41: 745-750. doi: 10.1161/ STROKEAHA.109.572065.

Rosewilliam S, Bucher C, Roffe C, Panyan A (2009) An approach to standardize, quantify and record progress of routine upper limb therapy for stroke subjects: the Action Medical Upper Limb Therapy protocol. Hand Therapy 14: 60-68.

Schulz KF, Altman DG, Moher D, CONSORT group (2010) CONSORT 2010 Statement: updated guidelines for reporting parallel group randomised trials. British Medical Journal 340: c332. doi: 10.1136/bmj.c332.

van Kuijk A, Pasman JW, Hendricks HT, Zwarts MJ, Geurts AC (2009) Predicting hand motor recovery in severe stroke: the role of motor evoked potentials in relation to early clinical assessment. Neurorehabilitation and Neural Repair 23: 45-51. doi: 10.1177/1545968308317578.

Wallace A, Talelli P, Dileone M, Oliver R, Ward N, Cloud G, Greenwood R, Di Lazzaro V, Rothwell JC, Marsden JF (2010) Standardizing the intensity of upper limb treatment in rehabilitation medicine. Clinical Rehabilitation 24: 471-478. doi: 10.1177/0269215509358944.

Whyte J, Dijkers M, Hart T, Zanca J, Packel A, Ferraro M, Tsaousides T (2014) Development of a theory-driven rehabilitation treatment taxonomy: conceptual issues. Archives of Physical Medicine and Rehabilitation 95: 2432. doi: 10.1016/j.apmr.2013.05.034.

World Health Organisation (2015) www.who.org [Accessed March 4 ${ }^{\text {th }}, 2015$ ].

World Stroke Organisation (2015) www.world-stroke.org/advocacy/worldstroke-campaign [Accessed March 6th, 2015]. 


\section{APPENDIX 1: DEFINITIONS OF UPPER LIMB REHABILITATION INTERVENTIONS}

1. Passive Interventions: Interventions that do not require patient participation and are performed by a therapist or therapy assistant.

- PROM / Ranging: These terms were considered to be interchangeable. This technique can be done in all positions (side lying, supine, standing). It entails taking individual joints of the UL through full available and pain free range passively (unassisted by patient). Passive movements aim to maintain joint ROM and muscle length. PROM/ranging is not commonly used as an isolated practice; it can be part of mobilisations and sensory re-training, eg finger PROM, 'scapula setting' and passive ranging of hand and wrist. PROM can also be done in proprioceptive neuromuscular facilitation (PNF) patterns and 'PNF ranging' was defined as passive movements in a PNF pattern which may or may not include verbal prompting.

- UL Stretch: is a sustained passive stretch to maintain joint ROM and muscle length. Stretches involve use of air splints, inhibition techniques to decrease overactivity, and manual stretching.

\section{- UL Mobilisations (trunk, scapula, hands and shoulder):}

- Scapular - both hands are placed on the scapula to mobilise it on the trunk e.g. movements of elevation, depression, protraction and retraction. This can involve movement of the scapula on trunk and trunk on scapula. Also documented as passive scapular mobilisations which are commonly incorporated in passive and active assisted ranging (either in supine, side lying, or sitting). This provides sensory input and aims to strengthen scapular movements leading to overall better UL movement and function. This may be performed during a functional task.

- Shoulder - anterior-posterior (AP) and caudad mobilisations of the glenohumeral head.

- Wrist - radio-ulnar, interphalangeal (IP) mobilisations.

- Hand - to mobilise joints when stiff and painful, also to increase sensory input, decrease tone, improve acceptance of base of support.

- UL positioning and C- cushion: Maintaining a good biomechanical alignment of the affected UL limb throughout the day. This may include using supports such as c-cushion, lap trays, shoulder sling, shoulder cuff and other equipment. It also entails patient, staff and family education regarding keeping the glenohumeral joint and all other joints of the UL in an optimal position. These strategies are predominantly used at rest when the patient is either in bed or sitting in a chair but not actively using the limb (outside of opportunities for functional use of the UL). However, they may also be used when the patient is eating meals, in the shower etc.
The Occupational Therapists noted that it is most likely to be documented as "positioning" in future.

- Shoulder Taping: for subluxed shoulder to increased glenohumeral stability in the presence of weak proximal muscles and to improve the alignment between the head of the humerus and the glenoid fossa. Taping can be used prior to facilitation to aid with normal movement patterns. In regards to shoulder subluxation management, some centres use a shoulder cuff support rather than providing taping.

- Splinting (Soft): aims to achieve stretch of muscles and maintain ROM of the joints and soft tissue length from hand to elbow, also to decrease tone. Often used at night time so it does not impair active movement. Also used to maintain skin integrity and facilitate hygiene.

- Thermoplastic splinting: to maintain joint integrity and muscle length via custom made thermoplastic splints. Soft splinting is mostly used for the management of hygiene issues in the presence of high tone (palm protectors, elbow splints).

2. Active-Assisted and Active Interventions: interventions that are facilitated by a therapist, or performed with supervision.

- UL Movement Facilitation: active-assisted exercise with the therapist using manual facilitatory techniques, such as muscle tapping, and modifying their input in response to patient's motor activity. This category may include joint compression, distraction at the shoulder, scapula, elbow, wrist and fingers with support as required in order to facilitate normal movement patterns. It may include verbal cues from therapist, external focus of control cues and directing the patient's visual attention to the affected limb.

- UL Facilitation of Fractionated Movement: facilitation of 3-joint movements usually starting with initiation in wrist extension (out of synergy). UL facilitation includes functional tasks such as reach and grasp practice, always with a functional goal or target. Positions may include supine, sitting or standing. Trunk constraints in sitting or standing may be used, including graviceptor activation. 'Hand on body' can also provide tactile feedback to the patient e.g. putting their own hand on their head. Other terms which may be used interchangeably: Facilitation of UL - exercise (hand on head), reaching practice in sitting with AP and lateral pelvic tilts (precursory to reaching activities).

- Reach and Grasp Practice/ Reaching facilitation: activeassisted movement through normal kinematic pattern for reach, grasp and release with modification of support in response to motor output. Manual handling given by the therapist includes sensory and proprioceptive input via auditory and tactile feedback through scapula, upper arm into elbow and wrist extension, supination, and finger extension then finger flexion to grasp. Should incorporate use of props and objects when possible and appropriate. Practice must be task specific and address a functional goal. 
The UL could be facilitated proximally or distally and against gravity, in accordance to the patient's deficit(s). For instance the UL could be facilitated to reach to the patient's knee or to a specific target in lying, sitting and standing. Therapists provided facilitation of trunk activation prior to the reaching task and guided reach task specific techniques. A number of therapists indicated use of 'trunk activation techniques' prior to reach and grasp tasks. Trunk Activation in particular refers to using Bobath strategies to augment truck control, weight bearing and weight shifting. Work on the pelvis and trunk is often incorporated into a functional task such as reaching - often starting proximally and working to improve distal control. This category may include seating review and provision of cushioning in the wheel chair to activate or dampen trunk activity as required. Also UL reaching activities to activate the trunk without any specific technique applied to the trunk. Trunk facilitation is commonly done to enable functional reach for an object outside the patient's base of support.

- Active Assisted Ranging / ROM: therapist provides facilitation/inhibition, in association with sensory and proprioceptive input and key points of control, working from the shoulder control down to fine wrist and hand movement. The whole body posture is also considered when promoting normal movement patterns. Remedial and compensatory approaches can include use of equipment. This task includes a functional and purposeful component such as reaching for a cup, grooming, feeding. Other terms that may be used interchangeably:

- Functional Reaching Facilitation and Active-assisted UL re-training. PNF movement facilitation: Active assisted facilitation as required using PNF patterns.

- Trunk Work/ Activation / Facilitation/ Alignment: Refers to the therapist's use of manual facilitation of selective activity of the trunk in order to gain/improve postural control. It could also incorporate selective activity of the upper limb on a stable trunk such as activation of side flexors in combination with reaching forward and leaning outside of base of support (i.e. internal and external displacement). Techniques commonly used in sitting include: lumbo-pelvic tilts, lateral and anterior-posterior pelvic tilt mobilisations, thoracic flexion and extension over a stable pelvis. This facilitation aims to disassociate trunk and pelvis via the thorax or central key points (CKP). Dissociation of CKP is also known as 'central key point (CKP) facilitation'. Therapists with more recent Bobath course attendance suggested that the term CKP is no longer current.

Proximal Stability:

- Scapular Facilitation / Setting: Also documented as Proximal Stability Exercises. Postural control must be achieved first prior to facilitating a stable/ set scapula. The affected hand is placed on a stable surface (contactile response); the therapist provides manual techniques in order to activate the scapular stabilisers. Proximal stability work can also be done in a weight bearing position in sitting.
- UL weight bearing exercises: weight bearing and weight shifting on the plinth or bolster, always on an extended arm. Also in side lying (side sitting or forward lean sitting), weight bearing through the arm for elbow, shoulder and scapula control. This allows setting of the scapula. UL weight bearing (hands on plinth): this refers to a position rather than a treatment technique.

\section{Interventions for Sensory and Proprioceptive Impairments:}

- Sensory \& Proprioceptive re-training / input: This includes providing opportunities for patients to increase their awareness of forms and location of sensory input to the body such as light touch, detecting sharpness, temperature, compression, traction, weight bearing exercises, massage, touching different textures, proprioception, visual and auditory input. Refers to any techniques used in order to tap into the sensory system. Sensory work to the hand particularly to the finger tips and movement creases i.e. distal palmar crease and thenar, hypothenar muscles and webspaces e.g. asking the patient to find these anatomical areas. Manual techniques include scratching/pricking (also pinch, prod, rub) applied to the finger tips e.g. thumb and index finger apposition and input to the palmar creases, finger and hand joint mobilisations (compression / distraction), passive movements of hand / fingers. Sensory stimulation can also be applied to the lateral aspect of the hand with joint approximation and compression techniques Other forms of sensory input may include face stimulation with a face cloth and visual attention to task (i.e. tracking with eyes and head turning), progressing to sensory input to the shoulder, elbow, wrist and fingers on the affected side. Scapula- mobilisations and sensory/proprioceptive feedback e.g. Tapping on the inferior scapular border. Proprioceptive input can also be applied to the wrist and triceps with joint mobilisations proximally and distally. Sensory retraining / input also includes patient and family education re: sensory input to UL.

Some therapists indicated that they use specific sensory retraining protocols, such as the Carey el al (2011) approach where possible; patients are encouraged to complete sensory discrimination and sensory stimulation tasks independently. Sensory retraining can be remedial (in the presence of specific deficits) or have the purpose of increasing attention to the affected upper limb also known as UL awareness i.e. "finding the hand".

- Stereognosis training: reaching into a bag and using tactile skills to recognise objects (stereognosis).

- Recognition of hot/cold, and sharp/ blunt input: These are more commonly used as an assessment tool and to inform type of sensory re-training required light touch versus sharp/blunt.

\section{Tone Management:}

- UL Releases/Mobilisations for Tone Management: a manual technique applied repetitively by the therapist which incorporates 'muscle releases' with distraction and a rotatory component in order to improve muscle length. 
This technique is applied slowly and is modified by the therapist according to patient's response i.e. muscle 'letting go' or increasing tone. This technique is documented as "mobilisation of muscles with rotation".

5. Oedema Management for the UL: Includes techniques such as neuromuscular electrical stimulation (NMES), bandaging, and use of compressive gloves, manual oedema mobilisation, and patient education on self-management. Massage and glove: using compressive gloves, retrograde massage, passive and active ranging, education for patients and family members. May include vibration in combination with elevation and positioning with c-cushions. Retrograde massage was defined as massaging the UL positioned above heart level, starting at a distal point (tips of fingers, wrist/forearm) towards the proximal aspects (shoulder) towards the heart.

\section{Functional Interventions:}

- ADL/ Functional Retraining: Includes specific training in personal care skills including those required for showering, dressing, eating, toileting, bed mobility, and domestic chores e.g. meal prep/ kitchen skills, laundry, showering, dressing, cognitive and perceptual re-training in community access, money management and leisure activities. Bilateral activities integrating both arms into ADLs, eg holding a jar with one hand and taking the lid off with the other hand or picking up a cup with both hands. Both remedial and compensatory approaches are utilised as indicated. This training may incorporate family members and education/ training. In some rehabilitation centres therapists may use the occupational therapy gym and a short stay functional training unit or the patient's home to provide a more realistic training environment. Graded discharges such as day leave or weekend leave are also considered part of ADL retraining and would be likely to involve task skill retraining in meaningful daily activities such as grooming, feeding, dressing, showering and toileting. For females it may encompass applying makeup, brushing hair and applying moisturiser. For men personal care tasks include brushing hair, shaving, brushing teeth and washing face.

- Fine motor skills practice: fine motor skills such as grasp, release, finger / thumb opposition, pincer grasp, facilitation of hand activity by working on intrinsic muscles of the hand and lumbrical muscle control. This category can include in hand manipulation of objects including props like beads, cards, nuts, bolts, buttons/ zips, and hand writing skill practice. Handwriting practice/ pen skills: with moderate to severe strokes this is likely to refer to training of writing/ pen skills with the unaffected upper limb as a compensatory strategy. For mild to mild-moderate impairments this is a graded process, series of handouts, templates. The use of different pen aids and surfaces may be incorporated as required. Functional tasks such as bimanual 'highly skilled' tasks like doing buttons up will also be included as appropriate. Also documented as "Dexterity exercises", however therapists prefer to call it "fine motor retraining". Fine motor skill practice tasks might require adequate alignment of the shoulder joint and scapular setting techniques.

\section{Patient \& Family Education:}

- Patient education regarding self PROM: usually involves teaching the patient to administer PROM using the unaffected hand to assist and passively move the affected hand/ limb.

- Self-Management of UL: this includes self PROM and education to increase safe self-management and handling of affected UL (e.g. prompting the use of cues such as "where is your arm?"). Specific instruction will be needed for oedema, ranging, positioning, implications of sensory loss, and inattention to avoid learned non-use and increase independence with ADLs. This category includes use of sensory kits and individualised programmes (eg for texture discrimination training). UL exercises (hand out): includes strengthening, coordination, positioning, and oedema management. Includes task specific exercises with functional outcomes.

- Hand exercises: strengthening by using theraputty, or resistance bands. Handouts outlining types of exercises are issued to patients. Exercises usually include opposition practice, isolation of finger movements and fine motor skills. Theraputty exercises: active finger movement against graded resistance for fine motor skills, strengthening of the hand muscles, sensory and proprioceptive input, bilateral tasks (simultaneously and alternating). Occupational Therapists issue an exercise sheet to patients.

- Family education re: sensory input and positioning: in regards to sensory input especially in the hand and arm, advice and guidance may be given to family including about: massage, pressure, scratching and light touch. Also advice would be provided reinforcing current management of the above and UL handling and positioning.

Additional interventions which participating therapists indicated were also used at their facilities:

- Visualisation / mental imagery or practice / guided imagery: a perceptual experience initiated by the patient, this could include mental imagery of a certain movement or functional task.

- Attention practice: encompasses getting the patient to attend to their affected UL via visual attention. This requires frequent prompting from the therapist. Visual constraint (covering unaffected UL with a towel).

- De-sensitization: use of sensory techniques described above to ameliorate oversensitive hand, forearm, or proximal UL.

- Mirror Box therapy: by using a box with a mirror on one side. The patient places the unaffected UL into outside of the box facing the mirror, and the affected UL in the inside of the box. The patient sees a reflection of the unaffected hand where the affected hand would be from an anatomical point of view. The patient completes a series of finger and wrist exercises at the same time as receiving 'artificial' visual feedback that the affected hand is now moving. Therapists indicated that patients complete a pre-mirror box activity such as right and left discrimination cards, and that at their 
facility mirror therapy is completed independently by the patient as adjunct to sessions. However, mirror therapy can also be completed as part of a rehabilitation session and some therapists are combining this with NMES.

- Neuromuscular electrical stimulation (NMES):

Facilitation of motor activity especially in shoulder, wrist and finger flexors and extensors. NMES is used to initiate and augment motor control; patients are encouraged to actively participate. Can be used in combination with the mirror box. Usually NMES is applied to the affected shoulder (over supraspinatus and posterior deltoid) to achieve glenohumeral joint re-alignment and improve subluxation or to wrist extensors to facilitate the initiation of reaching movements.

- Manual wheel chair training and positioning: this incorporates use of the unaffected upper limb and the affected limb as able. The task involves manoeuvring of wheelchair around the ward, community and even home.

- Electric Wheel Chair (EWC) Training: used as compensation for mobility and cognition. Also used for training neglect.

- Workshop: this term refers to a designated space with tools and experienced staff in wood and metal craft. The main aim is to integrate the upper limb, as a stabiliser or to manipulate tools, during bimanual tasks. Activities also involve cognitive planning.

\section{Reference:}

Carey L, Macdonell R, Matyas T (2011). SENSe: Study of the Effectiveness of Neurorehabilitation on Sensation - A Randomized Controlled Trial. Neurorehabilitation and Neural Repair 25(4):304-313.

\section{ACUTE \& SUB-ACUTE UPPER LIMB REHABILITATION}

\begin{tabular}{|l|l|l|}
\hline Pt's ID: & Session No: & \multirow{2}{*}{ Observations: } \\
\hline Date: & Location: & \\
\hline Time: & Subjective: \\
\hline Therapist: & \\
\hline
\end{tabular}

\section{List of Impairments:}

\section{Patient's Goals:}

\begin{tabular}{|c|c|c|c|}
\hline Passive Interventions & \multicolumn{2}{|l|}{ Time: } & \\
\hline $\begin{array}{l}\square \text { PROM / Ranging } \\
\square \text { PNF patterns "PNF ranging" } \\
\square \text { UL Stretch } \\
\text { Specify Joints / Muscles: }\end{array}$ & \multicolumn{3}{|c|}{$\begin{array}{l}\text { UL Mobilisations (Specify patient's position) } \\
\square \text { Trunk } \\
\square \text { scapula } \\
\square \text { Glenohumeral joint: AP or PA / Caudad } \\
\square \text { Hand / wrist: wrist, lumbricals, interosseous, MCPJ, ICPJ, thumb, radio-ulnar. } \\
\text { Comments: }\end{array}$} \\
\hline \multicolumn{3}{|l|}{ UL Positioning } & \\
\hline $\begin{array}{l}\square \text { Use of C-Cushion to maintain neutral } \\
\text { position }\end{array}$ & $\begin{array}{l}\square \text { Shoulder sling or hemi } \\
\text { cuff }\end{array}$ & $\square$ Lap Tray & $\square$ UL Trough \\
\hline
\end{tabular}




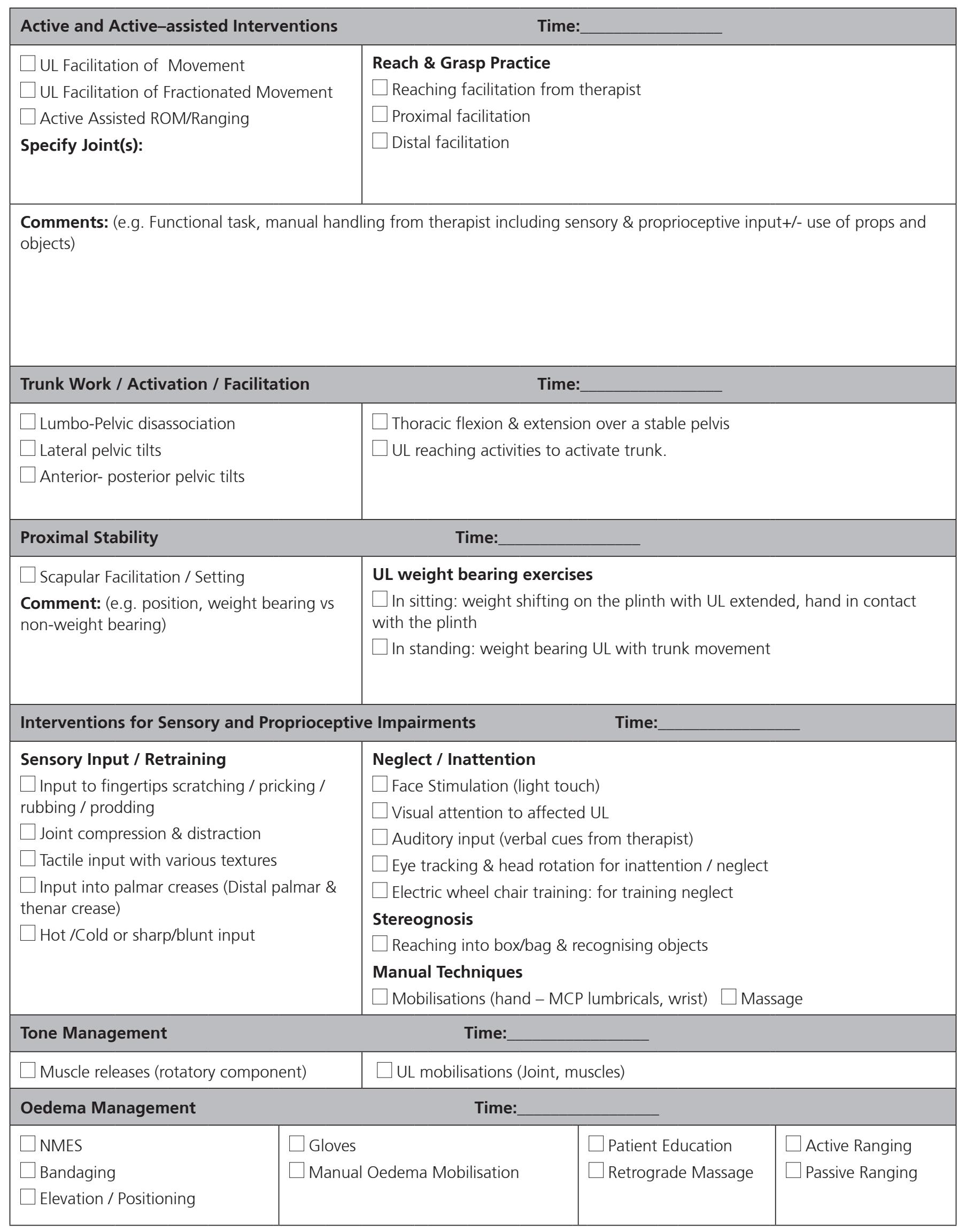




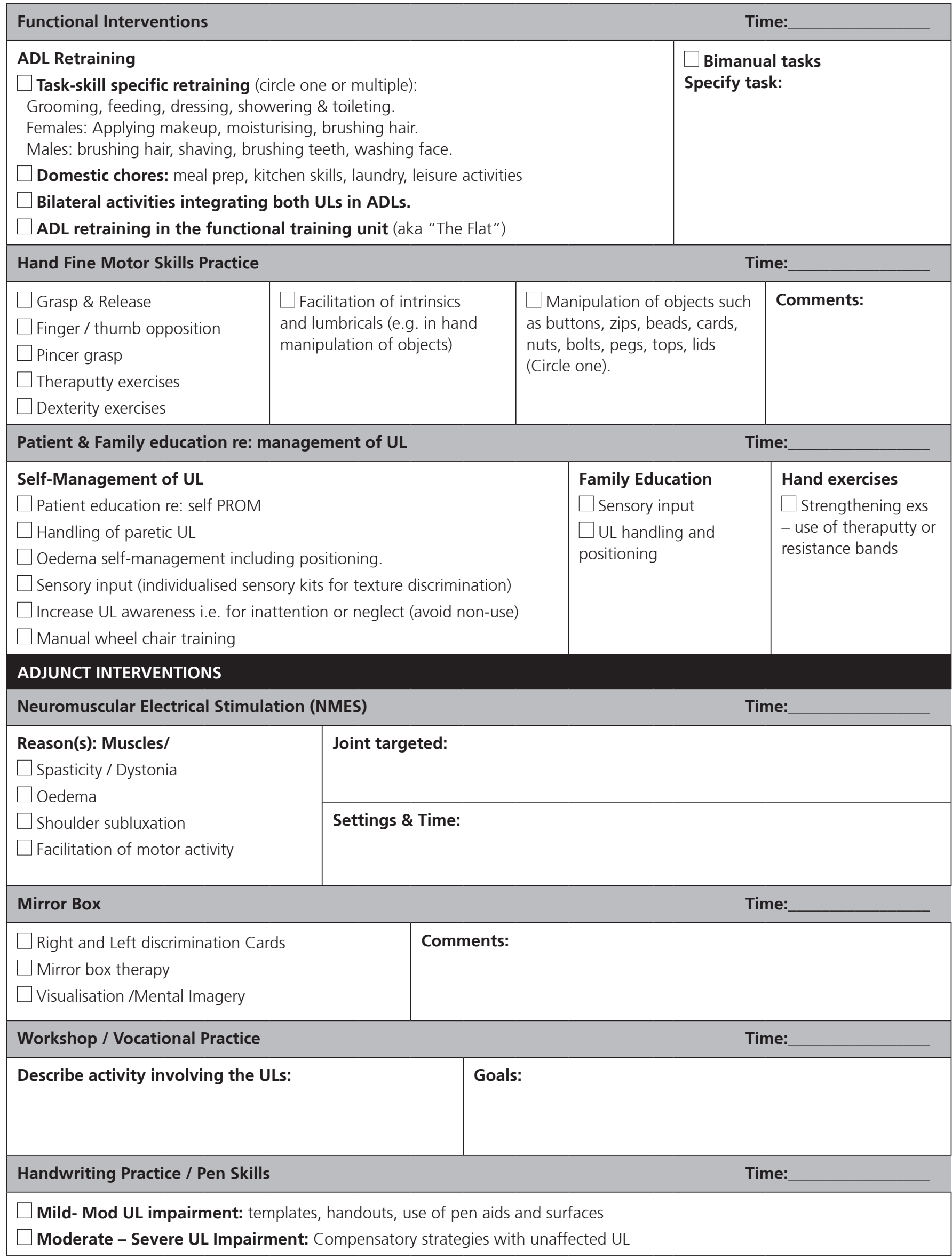




\begin{tabular}{|l|l|l|l|}
\hline Splinting & \multicolumn{1}{l|}{ Time: } \\
\hline $\begin{array}{l}\square \text { Thermoplastic } \\
\square \text { Soft }\end{array}$ & $\begin{array}{l}\text { Reason: } \\
\square \text { To maintain joint integrity } \\
\square \text { To maintain muscle length } \\
\square \text { To decrease tone i.e. palm protectors \& elbow splints }\end{array}$ & \multicolumn{1}{|c|}{ Comments: } \\
\hline Shoulder Taping & $\begin{array}{l}\square \text { For stability of weak } \\
\text { proximal muscles } \\
\text { subluxation }\end{array}$ & $\begin{array}{l}\square \text { Prior to UL facilitation to } \\
\text { assist with normal movement } \\
\text { patterns }\end{array}$ & Comments: \\
\hline
\end{tabular}

